\title{
Efficient coupling of photons to a single molecule and the observation of its resonance fluorescence
}

\author{
G. Wrigge, I. Gerhardt, J. Hwang, G. Zumofen, and V. Sandoghdar* \\ Laboratory of Physical Chemistry and optETH, \\ ETH Zurich, CH-8093 Zurich, Switzerland
}

\begin{abstract}
Single dye molecules at cryogenic temperatures display many spectroscopic phenomena known from free atoms and are thus promising candidates for fundamental quantum optical studies. However, the existing techniques for the detection of single molecules have either sacrificed the information on the coherence of the excited state or have been inefficient. Here we show that these problems can be addressed by focusing the excitation light near to the absorption cross section of a molecule. Our detection scheme allows us to explore resonance fluorescence over 9 orders of magnitude of excitation intensity and to separate its coherent and incoherent parts. In the strong excitation regime, we demonstrate the first observation of the Mollow triplet from a single solid-state emitter. Under weak excitation we report the detection of a single molecule with an incident power as faint as 150 attoWatt, paving the way for studying nonlinear effects with only a few photons.
\end{abstract}

\footnotetext{
*Electronic address: vahid.sandoghdar@ethz.ch
} 
Single dye molecules embedded in solids have been shown to behave as quantum mechanical two-level systems at cryogenic temperatures, exhibiting phenomena such as antibunching [1], AC Stark shift [2] and various nonlinear effects [3] known from atoms. The pioneering detection of single molecules was achieved via absorption spectroscopy assisted by a double lock-in method [4]. In the weak excitation regime, the effect of a plane-wave illumination on a molecule with a transition between levels $|1\rangle$ and $|2\rangle$ at wavelength $\lambda_{21}$ can be formulated as $I_{\mathrm{d}}=(1-\sigma / F) I_{\mathrm{e}}$. Here $I_{\mathrm{d}}$ is the detected intensity of the excitation beam after having encountered the emitter, $\sigma=3 \lambda_{21}^{2} / 2 \pi$ is the absorption cross section of the transition, and $F$ and $I_{e}$ are the area and intensity of the excitation beam, respectively. The experimental realization of this simple scenario is challenging because the effect of a single molecule has to be registered on top of the noise associated with a considerable laser power and scattering background. To improve the signal-to-noise ratio (SNR) in single molecule detection, fluorescence excitation spectroscopy was introduced [5]. In this technique the broad Stokes shifted fluorescence at $\lambda_{23}$ is detected on a very low background after filtering out light at $\lambda_{21}$ (see Fig. 1a). Unfortunately however, the impressive SNR of this method comes at the cost of losing the information on the coherences of the transition at $\lambda_{21}$.

Two recent reports have shown that it is also possible to detect single molecules coherently $[6,7]$ via interference between the excitation laser beam and the light scattered by the molecule. In the first scheme, the coherent scattering and a fraction of the reflected excitation beam were detected through a $10 \mu \mathrm{m}$ pinhole [6], resulting in a ratio of the order of $10^{-6}$ between the excitation and detected intensities. In the second experiment, cryogenic scanning near-field optical microscopy (SNOM) was employed to confine the excitation light to a subwavelength aperture with a typical transmission of $10^{-5}$ [7]. The great shortcoming of these methods is that more than 100,000 photons are required to detect one excitation. In view of quantum optical operations between photons and emitters, it would be highly desirable to approach a point where as many of the incident photons as possible interact with a single emitter. Such a regime would open the door to a wealth of nonlinear interactions between a single emitter and a single or few photons, which have been so far only achieved using sophisticated high finesse microcavities $[8,9,10]$. In this work, we show that it is possible to reach an efficient coupling between a single emitter and a freely propagating laser beam in a single-pass encounter if one reduces $F$ close to the diffraction limit [11]. 


\section{EFFICIENT FAR-FIELD DETECTION OF A SINGLE MOLECULE IN TRANS- MISSION}

A key technological hurdle in the study of single molecules in the condensed phase is to achieve a tight focus in the cryostat. Here we show that this problem can be addressed by exploiting the solid-immersion lens (SIL) technology [12]. As shown in Fig. 1b, a molded glass aspheric lens with $N A=0.68$ and a working distance of $1.76 \mathrm{~mm}$ focused the laser beam onto the sample, and a second identical lens was used to re-collimate it in the forward direction. A cubic zirconia half-ball lens with a diameter of $3 \mathrm{~mm}$ and a refractive index of about 2.18 was used as a SIL. Piezoelectrically driven slip-stick sliders were used to adjust the lateral and axial positions of the aspheric lenses with respect to the SIL. Fig. 1d shows
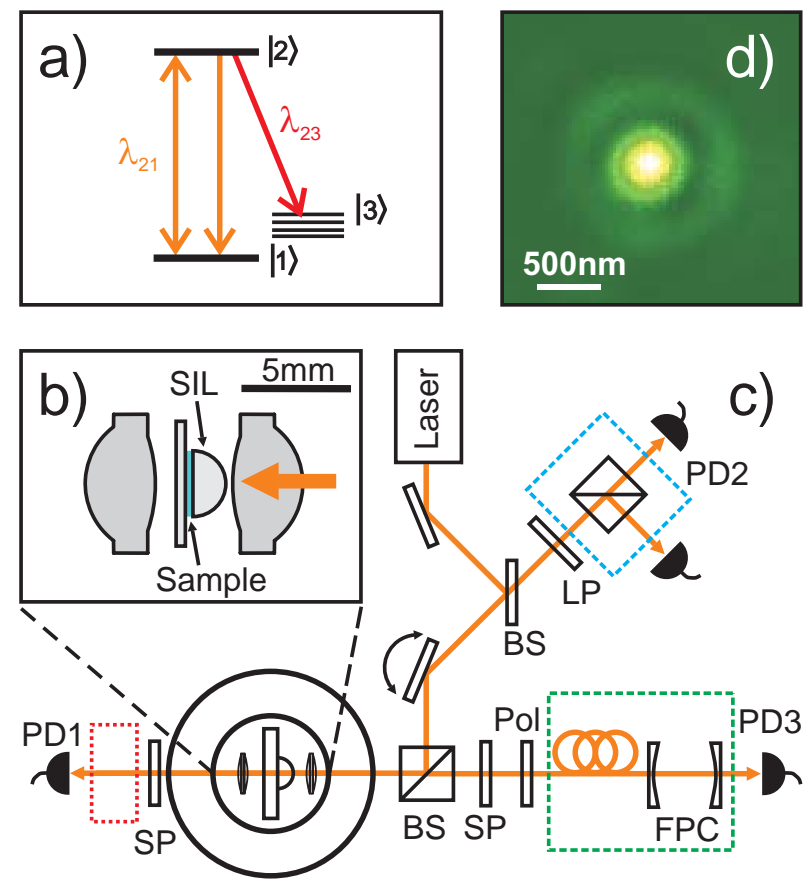

FIG. 1: a) The energy level scheme of a molecule with ground state $|1\rangle$ and excited state $|2\rangle$. Manifold $|3\rangle$ shows the vibrational levels of the electronic ground state. b) The arrangement of the lenses in the illumination and collection paths in the cryostat. c) The schematics of the optical setup. The dotted boxes are put in place for specific measurements discussed in the text. Abbreviations not explained in the text are BS: beam splitter, SP: short-pass filter, LP: long-pass filter, and Pol: polarizer. d) A laser scan image of a single molecule, showing a full width at half-maximum focus spot of about $370 \mathrm{~nm}$. 
a confocal fluorescence excitation scan of a single molecule. We achieved a focal spot with a full width at half-maximum of about $370 \mathrm{~nm}$ at $T=1.4 \mathrm{~K}$. Although this still does not meet the diffraction limit, it constitutes a great improvement over previous cryogenic far-field resolutions and allowed us to perform several new experiments, as described in the following.

Figure 1c sketches the experimental arrangement. A tunable dye laser with a linewidth of $1 \mathrm{MHz}$ was used to excite the molecules. The laser beam could be scanned across the sample with the aid of galvanometric mirrors. A Berek variable waveplate was used to precompensate for a slight elliptical polarization that was accumulated along the optical path and to ensure linear polarization at the position of the molecule. The samples consisted of a Shpol'skii matrix doped with dibenzanthanthrene (DBATT) molecules [13]. To prepare a sample, a small amount of DBATT was dissolved in $n$-tetradecane, applied to the flat side of the solid immersion lens and then squeezed and held in place by a glass cover slip. The 0-0 vibronic transition of DBATT in $n$-tetradecane is at a wavelength of $\lambda_{21} \sim 590 \mathrm{~nm}$. We routinely measured near to natural lifetime limited linewidths of $17-20 \mathrm{MHz}$ consistent with our independent measurements of $9.7 \mathrm{~ns}$ for the excited state fluorescence lifetime. Another favorable feature of DBATT is a very short triplet state lifetime [13].

Figure 1c shows three detection channels that were used for various measurements discussed in this article. The avalanche photodiode PD1 recorded the intensity of the transmitted light close to the excitation wavelength $\lambda_{21}$ by using a short-pass filter. The second detector (PD2) was situated after a long-pass filter and registered the Stokes shifted fluorescence on transitions $2 \rightarrow 3$. A third detector (PD3) was used in combination with other optical elements to record the resonance fluorescence intensity in reflection. In each experimental run, single molecules were first identified and characterised on PD2 using conventional fluorescence excitation spectroscopy. After optimization of the lateral focus position (see Fig. 1d), the polarization of the excitation light was aligned with the dipole moment of the molecule. The molecules that were selected for the experiments presented here showed no significant axial component in the orientation of their dipole moments and fluoresced with nearly perfect linear polarization.

Figure 2 shows an example of the raw transmission spectra recorded on PD1 with the above-mentioned approach. A dip of $11.5 \%$ is observed directly in transmission when a single molecule is resonant with the laser beam. The noise level amounts to $0.7 \%$, corresponding 
to the shot-noise limit of the incident intensity over an integration time of $160 \mathrm{~ms}$ per pixel. We emphasize that no noise suppression technique such as lock-in detection was employed in this work.

Although one might be inclined to attribute the observed dip to "absorption", the excitation spectrum $I_{\mathrm{d}}(\nu)$ registered on the detector has to take into account the interference between the excitation field $\mathbf{E}_{\mathrm{e}}$ and the field $\mathbf{E}_{\mathrm{m}}$ scattered by the molecule $[6,7]$ according to

$$
I_{\mathrm{d}}=\left\langle\widehat{\mathbf{E}}_{\mathrm{e}}^{-} \cdot \widehat{\mathbf{E}}_{\mathrm{e}}^{+}\right\rangle+\left\langle\widehat{\mathbf{E}}_{\mathrm{m}}^{-} \cdot \widehat{\mathbf{E}}_{\mathrm{m}}^{+}\right\rangle+2 \operatorname{Re}\left\{\left\langle\widehat{\mathbf{E}}_{\mathrm{e}}^{-} \cdot \widehat{\mathbf{E}}_{\mathrm{m}}^{+}\right\rangle\right\}
$$

In our recent work using SNOM [7, 14] we expressed the detector signal in detail, accounting for the modal evolution of the incident and scattered fields and for the spectroscopic properties of the molecule. Here we simplify the full expression from Ref. [7] in the form

$$
I_{\mathrm{d}}=I_{\mathrm{e}}\left[1+A \mathcal{L}(\nu)-B \mathcal{L}(\nu)\left(\Delta \cos \psi+\frac{\gamma}{2} \sin \psi\right)\right]
$$

where

$$
\mathcal{L}(\nu)=\frac{1}{\Delta^{2}+\gamma^{2} / 4+\Omega^{2}\left(\gamma / 2 \gamma_{0}\right)}
$$

is a Lorentzian line profile, and $\Delta$ denotes the laser frequency detuning. The quantities $\gamma$ and $\gamma_{0}$ stand for the full width at half-maximum values of the homogeneous and natural linewidths of the zero-phonon component of the 0-0 transition, respectively. The parameter $\Omega=\mathbf{E}_{\mathrm{e}} \cdot\langle 1|\mathbf{D}| 2\rangle / h$ is the Rabi frequency, where $\mathbf{D}$ signifies the dipole transition operator. $A$ and $B$ are positive coefficients whereas $\psi$ signifies the phase difference between the excitation and the coherently scattered light components.

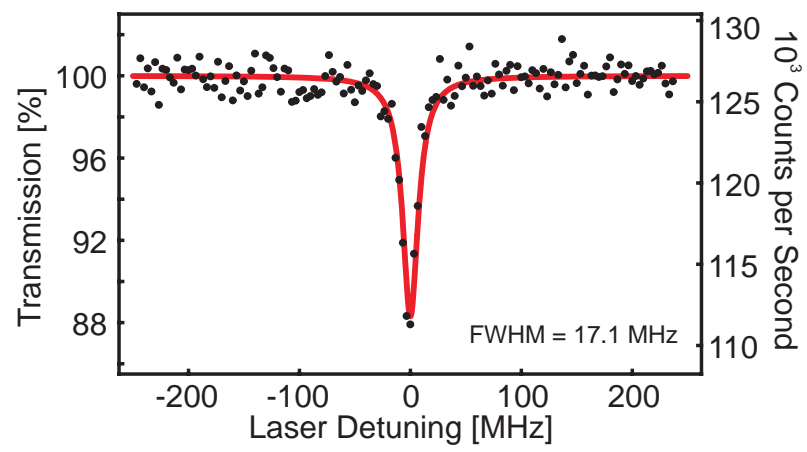

FIG. 2: An example of a raw transmission spectrum, revealing a $11.5 \%$ dip. The integration time per pixel was $160 \mathrm{~ms}$ and the noise amounts to $0.7 \%$. 
Equation (2) describes the spectrum that one registers on a detector as one scans the laser frequency. The first term is simply the part of the incident intensity that arrives at the detector. The second term proportional to $A$ represents the molecular emission and is always positive. In total, it consists of the narrow-band resonance fluorescence at $\lambda_{21}[15,16]$, the phonon wing of this transition caused by the vibrational coupling of the molecule with the matrix, and the Stokes-shifted fluorescence emitted at $\lambda_{23}$. In the signals detected on PD1 and PD3, however, the latter is filtered out. The third term in this equation proportional to $B$ denotes the interference between $\mathbf{E}_{\mathrm{e}}$ and the coherent part of $\mathbf{E}_{\mathrm{m}}$. In Refs. [7, 14], we showed various examples where $\psi$ could be varied, resulting in dispersive transmission spectra. In the weak excitation limit, the transmission signal on detector PD1 is dominated by the interference component, also known as the extinction term.

We emphasize that the observed interference effect depends on the mode overlap between the molecular emission and the incident beam and can be influenced by the choice of the detection solid angle. In our case, total internal reflection at the asymmetric geometry of the SIL-sample-glass-air layers can lead to a fine structure in the reflected beam [17] and take away energy from the transmitted beam. This arrangement also results in a strongly asymmetric molecular emission pattern which favors emission into the SIL side by about ten times [18]. Furthermore, for a given geometry the spectroscopic features of the emitter play an imporant role. The emission associated with the narrow-band zero-phonon line of the 0-0 vibronic transition is lowered by the Debye-Waller and Franck-Condon factors $\alpha_{D W}$ and $\alpha_{F C}$, which amount to 0.25 and 0.3 in our system [7], respectively. Thus, as compared to a perfect two-level system in the same geometric configuration, our molecule has had $1 /\left(\alpha_{D W} \alpha_{F C}\right) \sim 12$ times weaker coherent interaction with the laser beam.

\section{COHERENT RESONANCE FLUORESCENCE}

In addition to providing a strong detection signature, direct extinction spectroscopy offers the great advantage that it gives access to the narrow-band emission on the zero-phonon line. The radiation of a two-level system at the wavelength of the excited optical transition is known as resonance fluorescence. In case of weak resonant excitation and in the absence of dephasing, this emission is purely elastic and coherent $[15,16]$. As the incident

intensity is raised, this component first grows linearly, but it reaches a maximum at satura- 
tion parameter $S=\frac{\gamma \Omega^{2} / 2 \gamma_{0}}{\Delta^{2}+\gamma^{2} / 4}=1$. Under strong illumination, the population of the excited state undergoes Rabi oscillations at frequency $\Omega$ and the emitted field is contaminated by an inelastic incoherent component [16]. The coherent part of the resonance fluorescence is predicted to decrease slowly according to the text-book formula $S /(1+S)^{2}$, shown by the solid black curve in Fig. 3 .

The experimental assignment of the coherent and incoherent parts of resonance fluorescence is a nontrivial task because for a given excitation intensity, the detected spectrum contains both. The Lorentzian peak of the $A$-term in Eq. (2), receives contributions from both components. However, only the coherent emission can interfere with the incident light to produce the $B$-term, which is in turn composed of a Lorentzian part and a dispersive signal. Thus, one cannot decipher the variation of the coherent fluorescence with the excitation intensity in an unambiguous manner by simply monitoring the dip in Fig. 2. Below we show that it is possible to do this by influencing the individual components in a controlled manner.

As illustrated by Fig. 4a, for this experiment we have introduced a quarter waveplate and a polarizer in the detection path of PD1 at the position marked by the dotted red box in Fig. 1c. We set $\mathbf{E}_{\mathrm{e}}$ at $45^{\circ}$ with respect to the transition dipole moment of the molecule and at $80^{\circ}$ with respect to the axis of the polarizer. After traversing the quarter waveplate, the amplitudes and phases of $\mathbf{E}_{\mathrm{e}}$ and $\mathbf{E}_{\mathrm{m}}$ have evolved differently, thus changing the relative

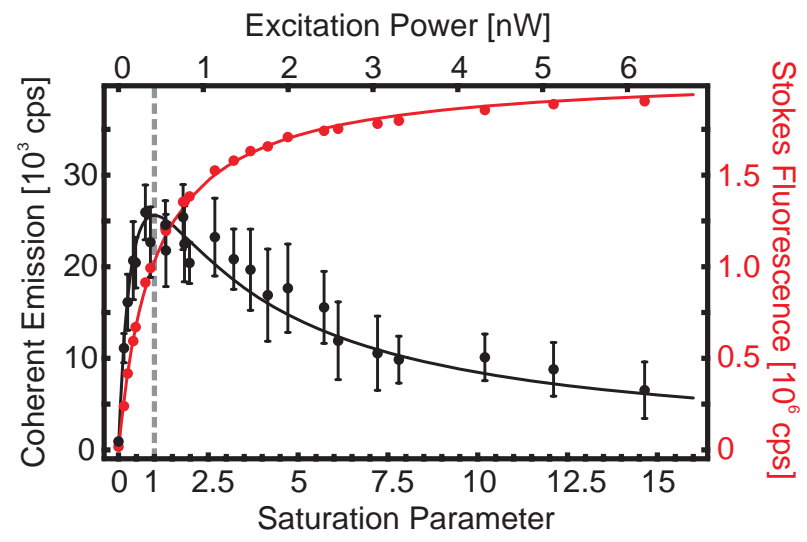

FIG. 3: Stokes shifted signal (red symbols) and the coherent part of the resonance fluorescence (black symbols) as a function of the laser excitation power, also expressed in terms of the saturation parameter $S$. The solid curves are the results of the theoretical calculations according to text-book formulae discussed in the text. 
phase $\psi$ and the coefficients $A$ and $B$. When rotating the quarter waveplate, both the strength and the shape of the $B$-Term in Eq. (2) are altered, but the Lorentzian peak of the $A$-term only changes its magnitude. By recording and analyzing a series of spectra, we were able to identify each component in a unique manner. The symbols in Figs. 4b and c display
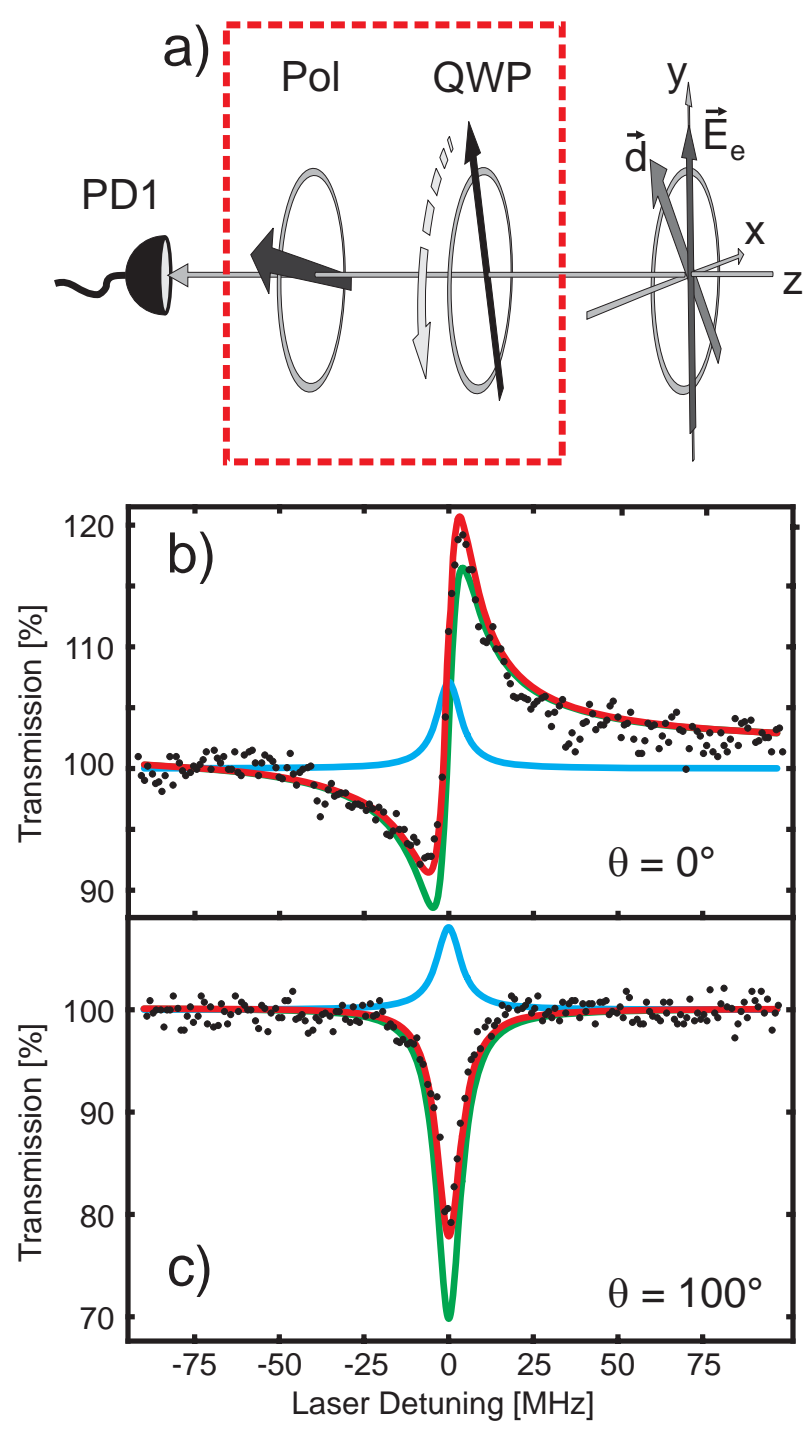

FIG. 4: a) Arrangement of a quarter waveplate (QWP) and a polarizer (Pol) for the separation of the coherent and incoherent parts of the resonance fluorescence. The laser field $\left(\mathbf{E}_{\mathrm{e}}\right)$ is taken to be along the y-axis whereas the molecular dipole moment (d) and the QWP are set at 45 and $\theta$ from this axis, respectively. b, c) Two examples of the recorded spectra are shown by the symbols. The red curves are the fits to the experimental data while the blue and the green lines plot the contributions of the molecular fluorescence intensity and extinction components respectively, as extracted from the series of measurements described in the text. 
two examples of such an analysis. The red curve is the fit to the experimentally recorded spectrum. The blue and the green spectra show the molecular fluorescence intensity and the extinction components that were extracted from the total signal, respectively. In case of Fig. 4b, the addition of the $A$-term has resulted in a final asymmetric dispersive shape whereas in Fig. 4c, the peak of $8 \%$ associated with the $A$-term has reduced a $30 \%$ dip of the $B$-term to yield a total observable dip of $22 \%$.

From the knowledge of the extinction $B$-term and $I_{\mathrm{d}}$ we can calculate the absolute number of the coherently scattered photons for a given excitation intensity. To measure the intensity dependence of the coherent resonance fluorescence, we have varied the excitation intensity between $5 \mathrm{pW}$ and $10 \mathrm{nW}$. The black symbols in Fig. 3 show the power of the coherent emission of a single molecule, extracted according to the procedure described above. We obtain an excellent agreement between the measured and the theoretical data (black solid curve) without using any free parameter. The red dots in Fig. 3 show the conventional saturation curve obtained from the fluorescence excitation signal recorded simultaneously on PD2 as a direct measure of the excited state population, also in excellent agreement with the function $S /(1+S)$ displayed by the solid red curve. We note that in our work $S$ was determined independently by analyzing the measured linewidths of fluorescence excitation spectra, whereby $S=1$ corresponds to a power broadened FWHM linewidth of $\sqrt{2} \gamma_{0}$.

The coherent part of resonance fluorescence has been previously put to evidence using a heterodyne measurement on the emission of a single ion [19]. However, to the best of our knowledge, a study of its intensity dependence presented in Fig. 3 has not been reported before. These measurements are particularly challenging in the solid state because of the omnipresence of background scattering at the excitation wavelength. The key to success in our experiment has been the efficient coupling of the laser light to the molecule, allowing us to optimize the ratio between the molecular signal and the background scattering. This is also nicely reflected in the fact that the onset of saturation at $S=1$ could be achieved at the very low incident laser power of $350 \mathrm{pW}$.

\section{MEASUREMENT OF THE MOLLOW TRIPLET}

The data in Fig. 3 clearly show that the coherent resonance fluorescence diminishes under strong excitation. This means that the interference between the laser beam and the 
molecular emission is reduced, and the dip in the transmission spectra recorded on PD1 gradually disappears. In parallel to this process, the inelastic component of the resonance fluorescence is expected to increase and give rise to a triplet spectrum as first described by B. Mollow in 1969 [20]. The so-called Mollow triplet has been indeed examined in various gaseous systems [21, 22], but its direct observation has turned out to be elusive in the solid state.

To record and study the incoherent part of the spectrum, we used the detection path in reflection that leads to PD3 in Fig. 1c. The fluorescence was sent through a short-pass filter to cut out the Stokes shifted fluorescence corresponding to the $2 \rightarrow 3$ transitions, while transmitting light at $\lambda_{21}$ and part of the broad phonon wing of the zero phonon line. Here the angle between $\mathbf{E}_{\mathrm{e}}$ and that of the molecular dipole was set to $45^{\circ}$ and the analyzing polarizer was aligned at $90^{\circ}$, filtering out the background scattering of the laser light with a ratio of 300:1. The remaining fluorescence was coupled into a multimode optical fiber with a core diameter of $50 \mu \mathrm{m}$ for convenience of handling. The output of the fiber was sent to a homebuilt Fabry-Perot cavity (FPC) with a free spectral range of $356 \mathrm{MHz}$ and transmission of approximately 15\%. As shown by the lowest spectrum in Fig. 5a, an instrumental linewidth of about $14 \mathrm{MHz}$ was measured when the reflected laser light was coupled to the resonator. Despite polarization filtering of the fluorescence, the background laser scattering was of the same order of magnitude as the molecular fluorescence and was exploited for calibrating the frequency axis of each FPC scan.

Figures 5b-h show a series of emission spectra recorded by exciting a molecule on resonance, collecting its fluorescence close to the same wavelength in the fashion described above and then scanning the FPC several times, equivalent to an integration time of $3 \mathrm{~s}$ per scan pixel. As the excitation intensity was raised (moving upward in the figure), side bands appeared in the molecular emission spectrum with increasing frequency separations. The appearance of the Mollow triplet can be understood as the result of the periodic modulation of the density matrix elements and thus the molecular polarization at the Rabi frequency.

To obtain an independent measure of the Rabi frequency, we split the Stokes-shifted fluorescence beam on PD2 via a beam splitter onto two photodetectors as sketched in the blue dotted box in Fig. 1c. By using the common start-stop scheme [23], we recorded the intensity correlation function $g^{(2)}(\tau)[15]$ for each measurement presented in Figs. 5b-h. The results are plotted in Figs. 5j-p. Figure 5i confirms that as expected, we observed pure antibunching 
at low intensities. However, as the excitation was made stronger, $g^{(2)}(\tau)$ underwent oscilla-

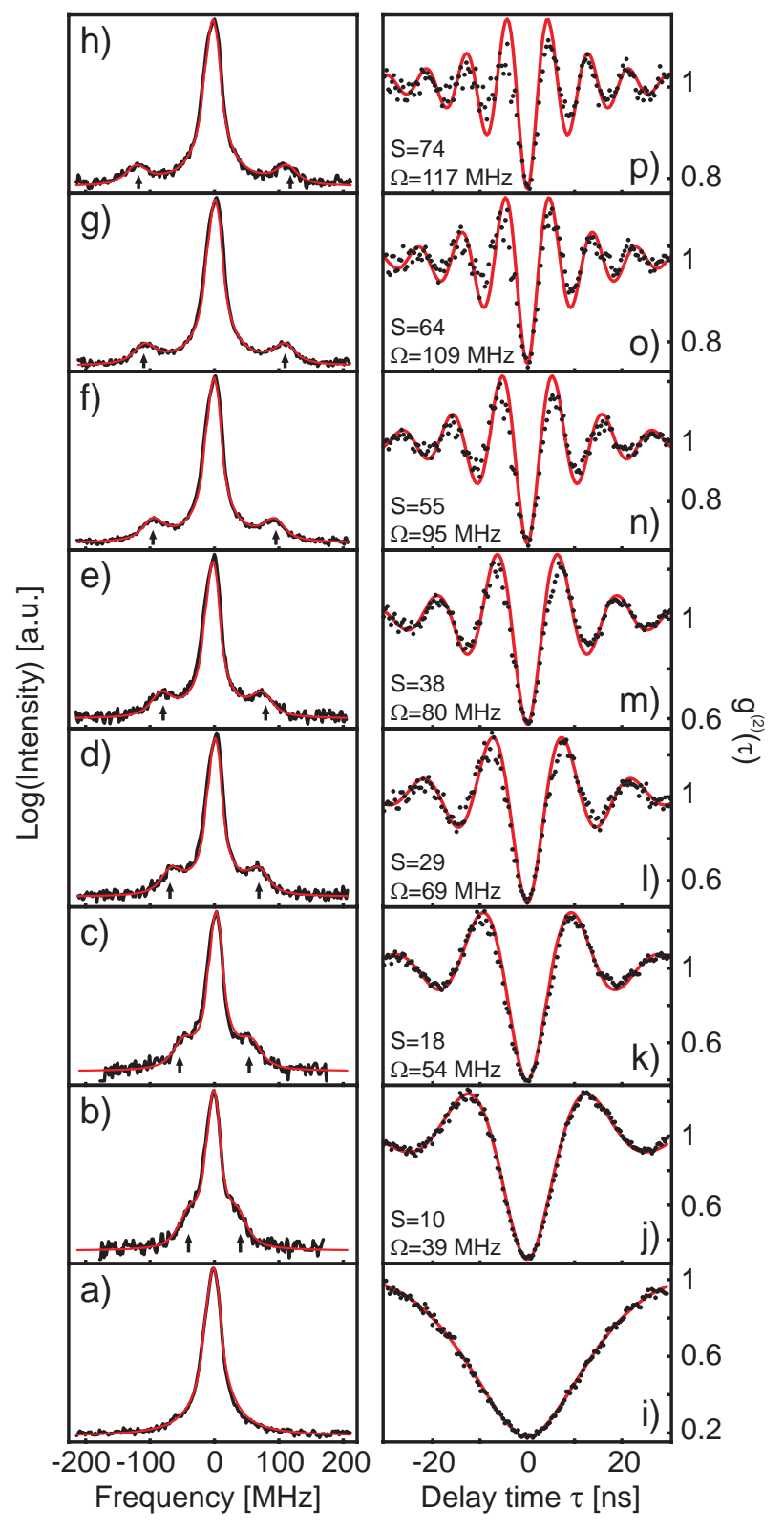

FIG. 5: a) Transmission of the FPC under laser illumination. b-h) Series of resonance fluorescence emission spectra (black) recorded by scanning the FPC for increasing excitation intensities. The red curves plot the calculated spectra. i) Second-order autocorrelation function of the single molecule under weak excitation, displaying photon antibunching. j-p) Series of autocorrelation functions recorded at the same time as the spectra shown in b-h). The Rabi frequency $\Omega$ extracted from the fit (red curves) to the $g^{(2)}(\tau)$ data is indicated in each figure together with the corresponding saturation parameter $S$. 
tions [1]. By fitting the curves in Figs. 5i-p, we determined the Rabi frequencies $\Omega$ in each case. These values together with the corresponding saturation parameters $S$ are indicated in Figs. 5j-p.

Using the independently measured values of $\Omega$, the laser background and the FPC line profile (see Fig. 5a), we then calculated the theoretical spectra for the Mollow triplet shown by the red curves in Figs. 5b-h. The agreement between theory and experiment is excellent with only one scaling parameter for the signal intensity. To our knowledge, this constitutes the first observation of the Mollow triplet from a single emitter in the solid state, further confirming the potential of these systems for well-understood and controlled quantum optical experiments.

\section{SINGLE MOLECULE DETECTION WITH ULTRA-FAINT LIGHT SOURCES}

Commonly a high flux of photons is required in spectroscopy to achieve a single excitation because either the observed transitions are strongly broadened or the light beam is focused very weakly. Moreover, a certain minimum excitation rate is required to obtain fluorescence count rates larger than the square root of the detector noise level. A remarkable advantage of coherent detection of single molecules, however, is that the detected intensity can easily be larger than the detector noise even if the molecular emission is much weaker. This is illustrated in Fig. 6 where a single molecule is detected under an incident power of merely 550 photons per second, equivalent to 150 attoWatt. The transmission spectrum shows the raw data integrated over 4 seconds at each pixel. When analyzing this result in the context

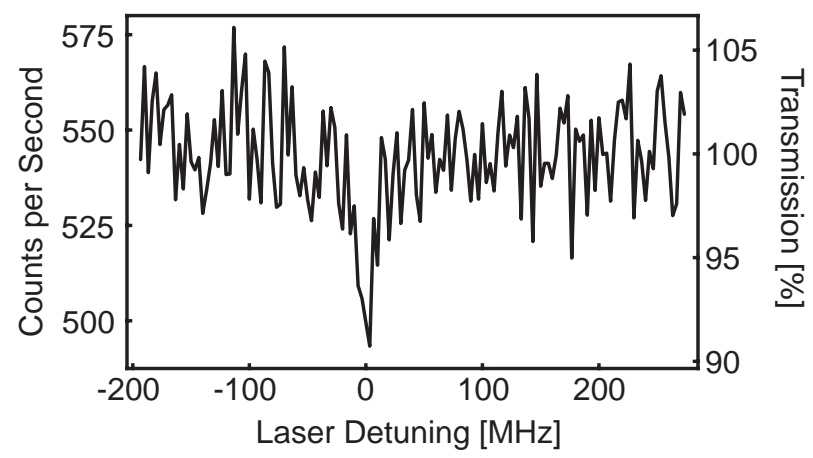

FIG. 6: An extinction spectrum recorded from a single molecule under an ultra-faint excitation power of 550 photons per second. 
of an interference phenomenon, we find that the dip of 50 counts per second originates from a detected molecular emission of only 1.1 photons per second, which is about 10 times lower than the noise associated with the 150 dark counts of our detector. Thus, the high signal contrast of coherent extinction measurements is ideally suited for detecting weak emitters such as single rare earth ions, especially if one would exploit additional improvements in the SNR via lock-in detection.

The ability to detect a single emitter with an ultra-faint illumination also shows the feasibility of exciting a single molecule by the emission of a second molecule acting as a source of Fourier-limited single photons [24]. Considering that the fluorescence of a single molecule typically amounts to about $10^{5}$ detected photons per second, we can easily expect a few thousands of photons per second at the position of the second molecule after various diagnostics and handling optics. Indeed, calculations show that in the case where an emitter is illuminated by a single photon Gaussian wavepacket of width $\gamma_{0}$, there is a higher than $50 \%$ chance for it to act as a $\pi$-pulse to coherently transfer the population of the ground state to the excited state [25] or to experience a substantial phase shift via its interaction with the molecule. Furthermore, it has been suggested that the molecule can act as a nonlinear medium to couple two ultra-faint photon fields impinging on it at the same time [25].

\section{CONCLUSION}

By confining a laser beam close to the diffraction limit at $\mathrm{T}=1.4 \mathrm{~K}$, we have examined for the first time, the coherent interaction of a single organic molecule with light over 9 orders of magnitude of excitation power. In the weak excitation regime, we have achieved a large extinction dip of $11.5 \%$ and have demonstrated the detection of a single molecule with an ultra-faint laser source of 150 attoWatt. In the strong excitation regime, our measurements have allowed us to distinguish the coherent and incoherent parts of resonance fluorescence and to study their intensity dependencies. Furthermore, we could directly record the Mollow triplet as a landmark of the nonlinear interactions between an emitter and laser light.

From a fundamental point of view, an intriguing question that arises is whether one could achieve a perfect coupling between a freely propagating optical mode and a dipolar emitter in a single-pass encounter, i.e without high-finesse cavities. When considering the interference nature of the extinction signal, it is clear that the annihilation of a photon 
in the forward direction requires perfect overlap between its mode and that of a dipolar radiator. Clearly, this condition is not satisfied for a strongly focused $\mathrm{TEM}_{00}$ laser beam [11]. However, it has been proposed that the combination of an emitter with an axially oriented transition dipole moment [26] and light with a radially polarized doughnut mode would be favorable $[27,28]$. Another exciting possibility might be to use nano-antennae for engineering the radiation pattern of the molecule and matching it to the excitation mode [29]. Given the achievements presented in this article and the simplicity of the SIL technology, we anticipate that our approach opens doors to the realization of these ideas and a wide range of other investigations on the coherent and nonlinear interaction of light with single molecules and ions in the condensed phase.

We are grateful to A. Renn for experimental support. This work was financed by the Schweizerische Nationalfond and the ETH Zurich initiative for Quantum Systems for Information Technology (QSIT).

The authors declare that they have no competing financial interests.

Correspondence and request for materials should be addressed to V.S. (email: vahid.sandoghdar@ethz.ch).

[1] Basché, T., Moerner, W. E., Orrit, M. \& Talon, H. Photon antibunching in the fluorescence of a single dye molecule trapped in a solid. Phys. Rev. Lett. 69, 1514-1517 (1992).

[2] Tamarat, P. et al. Pump-probe experiments with a single molecule: ac-stark effect and nonlinear optical response. Phys. Rev. Lett. 75, 1514-1518 (1995).

[3] Tamarat, P. et al. Non-linear optical response of single molecules. Chem. Phys. 245, 121-132 (1999).

[4] Moerner, W. E. \& Kador, L. Optical detection and spectroscopy of single molecules in a solid. Phys. Rev. Lett. 62, 2535-2538 (1989).

[5] Orrit, M. \& Bernard, J. Single pentacene molecules detected by fluorescence excitation in a p-terphenyl crystal. Phys. Rev. Lett. 65, 2716-2719 (1990).

[6] Plakhotnik, T. \& Palm, V. Interferometric signatures of single molecules. Phys. Rev. Lett. 87, 183602 (2001).

[7] Gerhardt, I. et al. Strong extinction of a laser beam by a single molecule. Phys. Rev. Lett. 
98, 033601 (2007).

[8] Turchette, Q. A., Hood, C. J., Lange, W., Mabuchi, H. \& Kimble, H. J. Measurement of conditional phase shifts for quantum logic. Phys. Rev. Lett. 75, 4710-4713 (1995).

[9] Hijlkema, M. et al. A single-photon server with just one atom. Nature Phys. 3, 253 - 255 (2007).

[10] Press, D. et al. Photon antibunching from a single quantum-dot-microcavity system in the strong coupling regime. Phys. Rev. Lett. 98, 117402 (2007).

[11] van Enk, S. J. \& Kimble, H. J. Strongly focused light beams interacting with single atoms in free space. Phys. Rev. A 63, 023809 (2001).

[12] Mansfield, S. M. \& Kino, G. S. Solid immersion microscope. Appl. Phys. Lett. 57, 2615-2616 (1990).

[13] Boiron, A.-M., Lounis, B. \& Orrit, M. Single molecules of dibenzanthanthrene in $n$ hexadecane. J. Chem. Phys. 105, 3969 (1996).

[14] Gerhardt, I. et al. Scanning near-field optical coherent spectroscopy of single molecules at 1.4 kelvin. Opt. Lett. 32, 1420-1422 (2007).

[15] Loudon, R. Quantum Theory of Light (Oxford University Press, 2000).

[16] Cohen-Tannoudji, C., Dupont-Roc, J. \& Grynberg, G. Atom-Photon Interactions (John Wiley \& Sons, Inc., 1992).

[17] Novotny, L., Grober, R. D. \& Karrai, K. Reflected image of a strongly focused spot. Opt. Lett. 26, 789-791 (2001).

[18] Koyama, K., Yoshita, M., Baba, M., Suemoto, T. \& Akiyama, H. High collection efficiency in fluorescence microscopy with a solid immersion lens. Appl. Phys. Lett. 75, 1667-1669 (1999).

[19] Höffges, J. T., Baldauf, H. W., Lange, W. \& Walther, H. Heterodyne measurement of the resonance fluorescence of a single ion. J. Mod. Opt. 44, 1999-2010 (1997).

[20] Mollow, B. R. Power spectrum of light scattered by two-level systems. Phys. Rev. 188, 1969-1975 (1969).

[21] Wu, F. Y., Grove, R. E. \& Ezekiel, S. Investigation of the spectrum of resonance fluorescence induced by a monochromatic field. Phys. Rev. Lett. 35, 1426-1429 (1975).

[22] Walther, H. Resonance fluorescence of two-level atoms. In Advances in Atomic, Molecular and Optical Physics, Vol. 51, 239-272 (Elsevier, 2005).

[23] Hanbury Brown, R. \& Twiss, R. Q. Correlation between photons in two coherent beams of 
light. Nature 177, 27-29 (1956).

[24] Kiraz, A., Ehrl, M., Bräuchle, C. \& Zumbusch, A. Ultralong coherence times in the purely electronic zero-phonon line emission of single molecules. Appl. Phys. Lett. 85, 920-922 (2004).

[25] Domokos, P., Horak, P. \& Ritsch, H. Quantum description of light-pulse scattering on a single atom in waveguides. Phys. Rev. A. 65, 033832 (2002).

[26] Pfab, R. J. et al. Aligned terrylene molecules in a spin-coated ultrathin crystalline film of p-terphenyl. Chem. Phys. Lett. 387, 490 (2004).

[27] Dorn, R., Quabis, S. \& Leuchs, G. Sharper focus for a radially polarized light beam. Phys. Rev. Lett. 91, 233901 (2003).

[28] van Enk, S. J. Atoms, dipole waves, and strongly focused light beams. Phys. Rev. A 69, 043813 (2004).

[29] Alù, A. \& Engheta, N. Enhanced directivity from sub-wavelength infrared/optical nanoantennas loaded with plasmonic materials or metamaterials. IEEE Trans. Antennas and Propagation in press (2007). 